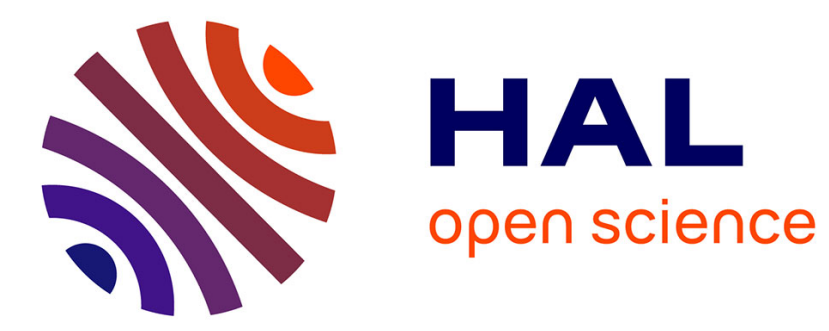

\title{
Sur les effets de mirage et les différences de densités qu'on observe dans les tubes de Natterer
}

P. Villard

\section{To cite this version:}

P. Villard. Sur les effets de mirage et les différences de densités qu'on observe dans les tubes de Natterer. J. Phys. Theor. Appl., 1896, 5 (1), pp.257-266. 10.1051/jphystap:018960050025700 . jpa-00239875

\section{HAL Id: jpa-00239875 https://hal.science/jpa-00239875}

Submitted on 1 Jan 1896

HAL is a multi-disciplinary open access archive for the deposit and dissemination of scientific research documents, whether they are published or not. The documents may come from teaching and research institutions in France or abroad, or from public or private research centers.
L'archive ouverte pluridisciplinaire HAL, est destinée au dépôt et à la diffusion de documents scientifiques de niveau recherche, publiés ou non, émanant des établissements d'enseignement et de recherche français ou étrangers, des laboratoires publics ou privés. 


\section{SUR LES EFFETS DE MIRAGE ET LES DIFFÉRENGES DE DENSITÉS QU'ON OBSERVE DANS LES TUBES DE NATTERER;}

Par M. P. VILLARD.

Quand on élève progressivement la température d'un tube de Natterer convenablement rempli, le ménisque, au lieu de disparaître nettement quand on franchit le point critique, fait place à une couche de transition. D'une extrémité à l'autre du tube l'indice et la densité varient alors d'une manière continue, mais la masse fluide ne devient pas, en général, homogène, même si l'on dépasse de plusieurs degrés la température critique.

De plus, comme l'a fait remarquer M. Pellat ('), on peut suivre le niveau jusqu'au point critique avec des tubes très diversement remplis.

M. Gouy $\left({ }^{2}\right)$ a, il est vrai, montré qu'en se plaçant dans de bonnes conditions et agitant fréquemment les tubes, on se rapprochait des résultats prévus par la théorie d'Andrews; en particulier, le niveau n'est alors observable jusqu'au point critique que si le remplissage est compris entre des limites que la théorie précédente permet de calculer en tenant compte de l'influence inévitable de la pesanteur.

J'ai, d'autre part établi qu'il était possible et probablement normal d'obtenir un fluide homogène, dans les tubes de Natterer, à partir de la température critique $\left({ }^{3}\right)$.

Mais les phénomènes signalés plus haut n'en restent pas moins à expliquer. Bien qu'ils soient dus en partie à l'influence de gaz étrangers, tels que l'air, cette cause perturbatrice, que j'ai déjà signalée (loc. cit.), est tout à fait secondaire.

Pour pouvoir étudier de près ce qui se passe dans un tube de Natterer pendant qu'on l'échauffe, j'ai fait construire par M. Chabaud l'appareil représenté ( $f g .1$ ), composé d'un tube de 8 millimètres de diamètre intérieur sur 20 centimètres de longueur, auquel sont soudés deux thermomètres disposés comme l'indique la figure. Un tube latéral $a$ sert à effectuer le remplissage et est ensuite fermé à la lampe.

(1) Journal de Physique, 3 série, t. I, p. 225 ; 1892.

(2) Comptes xendus, t. CXV, p. 720, t. CXVI, p. 1289, t. CXXI, p. 201.

(3) Journal de Physique, $3^{\circ}$ série, t. III; 1894. 
Cette disposition permet de suivre la marche des températures du liquide et de sa vapeur.

Le gaz employé a été l'éthylène préparé par l'action de l'anhydride borique sur l'alcool (Ebelmen) ( $\left.{ }^{1}\right)$, et purifié suivant la méthode générale déjà indiquée (loc. cit.).

La température critique de ce gaz étant voisine de $+10^{\circ}$ pouvait

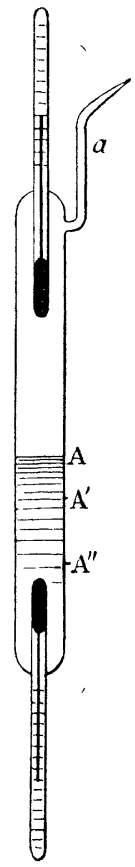

Fig. 1.

être facilement maintenue constante, ainsi que les températures un peu supérieures.

Le tube a été rempli de telle sorte qu'à $+9^{\circ}, 25$ tout le liquide fût vaporisé, quand on avait soin d'agiter le tube. De cette manière, aucune incertitude ne subsistait relativement à l'état physique de l'éthylène au voisinage immédiat du point critique.

La correction de pression relative aux thermomètres a été évaluée

(1) L'éthylène préparé par l'acide sulfurique et l'alcool ou l'éther est généralement impur, liquéfié : puis évaporé à $-80^{\circ}$, il bout à la fin de l'opération comme un liquide visqueux et laisse un résidu liquide peu volatil à $0^{\circ}$ et sirupeux à $-80^{\circ}$. Il n'en est plus de mème quand on emploie l'anhydride borique. 
en maintenant l'appareil à des températures connues $\left(6^{\circ},+10^{\circ},+10^{\circ}\right)$ et notant les indications des instruments quand elles étaient devenues invariables.

Les expériences suivantes ont été ensuite effectuées :

I. - L'appareil a été refroidi à $0^{\circ}$, puis plongé dans un bain d'eau de 12 litres, maintenu à $+11^{\circ}$ et agité par un courant d'air.

Ainsi que cela se passe en général, le niveau du liquide, situé en A, vers le milieu du tube, est resté sensiblement fixe, puis s'est transformé en une couche de transition. Ce n'est qu'au bout de plus d'une demi-heure que la masse fluide est devenue homogène.

Pendant ce temps, les indications des thermomètres, concordantes au début, se sont progressivement écartées de $\mathbf{1}^{\circ}$ environ, puis se sont rapprochées lentement pour redevenir concordantes au bout de plus d'une demi-heure. Un tube semblable au précédent, mais ne contenant que de l'eau, prend, au contraire, en six à sept minutes la température du bain.

Dans l'air à $+18^{\circ}$, le réchauffement de l'appareil donne lieu à un écart encore plus grand, soit $3^{\circ}$, entre les températures des deux thermomètres, et cet écart ne s'annule qu'au bout de plusieurs heures.

II. - Pour éviter toute erreur provenant d'un fonctionnement irrégulier des thermomètres, le tube a été retourné bout pour bout pendant que les thermomètres étaient en désaccord, mais au-dessus de la température critique. La masse fluide dense qui entourait le thermomètre froid est descendue sur le thermomètre chaud, le faisant baisser instantanément de plus d'un demi-degré.

III. - Pour pouvoir placer le réservoir d'un thermomètre près du niveau, il n'a pas été nécessaire d'employer un second appareil ; il a suffi d'utiliser une propriété des tubes de Natterer étudiée déjà par M. Gouy.

Dans l'appareil précédent, tout le liquide est vaporisé à $+9^{\circ}, 25$, si on a soin d'agiter le tube pour égaliser la température ; à $+9^{\circ}, 25$, le niveau atteint l'extrémité inférieure du tube; entre $0^{\circ}$ et $+9^{\circ}, 25$, il occupe une série de positions $\mathrm{A}, \mathrm{A}^{\prime}, \mathrm{A}^{\prime \prime}$, et peut se maintenir en chacune d'elles, jusqu'à sa disparition, si on continue à élever la température en cessant d'agiter. Le tube étant alors porté dans le bain d'eau $\grave{a}+11^{\circ}$ et maintenu immobile, les thermomètres n'ont pas tardé à être en désaccord, l'écart maximum entre leurs indications étant - presque aussi grand que dans l'expérience I. 
IV. - La simple observation du tube pendant l'échauffement permet de constater que la température est à peu près uniforme, d'une part, dans la masse liquide, d'autre part, dans la vapeur. En effet, dans la partie occupée par le liquide, les génératrices paraissent rectilignes, ce qui montre que l'indice est constant; il en est évidemment de même de la densité et de la température. Les génératrices ne commencent à s'infléchir que très près du niveau, et cela quand on approche de la température critique. Au-dessus du niveau, les génératrices sont infléchies sur une faible hauteur, puis redeviennent rectilignes, indiquant ainsi que la température redevient uniforme.

On voit, d'ailleurs, se former peu à peu au-dessus du niveau une couche de vapeur dense, surtout au voisinage du point critique. Par

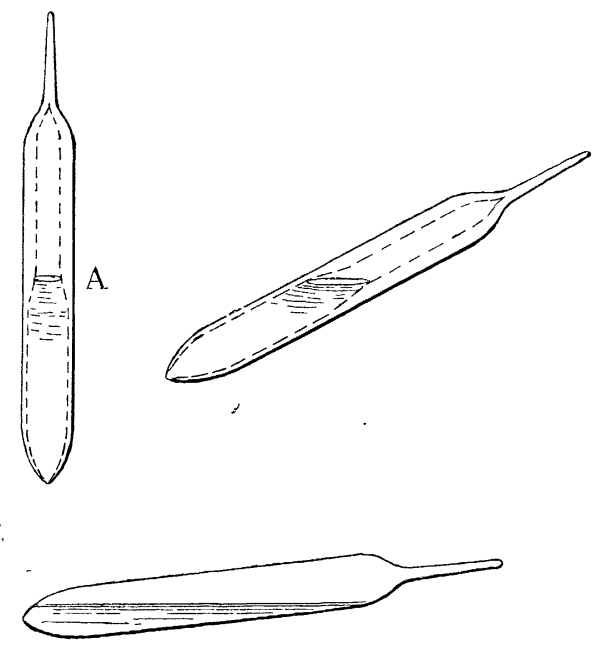

FIG. 2.

une agitation légère cette couche donne naissance à des stries ondoyantes.

$\mathrm{Au}$-dessus de la température critique c'est également là où a disparu le niveau que la température varie le plus rapidement avec la hauteur.

V. - Dans toutes les expériences, une fois la température critique dépassée, le contenu du tube est homogène, si les thermomètres sont d'accord, hétérogène dans le cas contraire. Remarquons en passant que cette observation est relative à un gaz très pur. 
VI. - Avec le tube précédent, ainsi qu'avec d'autres tubes diversement remplis, l'hétérogénéité du contenu peut être obtenue artificiellement, au-dessus de la température critique, en produisant une différence de température entre les deux moitiés du tube. Celui-ci ayant été conservé pendant plusieurs jours ou même plusieurs mois au-dessus de la température critique, à $+18^{\circ}$ par exemple, la moitié inférieure du tube est plongée dans de l'eau à $+12^{\circ}$ (soit $2^{\circ}$ au-dessus du point critique), la moitié supérieure du tube est tenue à la main. On voit alors ruisseler le long des parois le gaz contracté par refroidissement, comme on verrait de l'air s'élever le long d'une paroi échauffée. Au bout d'une demi-minute on peut retirer le tube: les génératrices offrent alors l'aspect représenté $f g$. 2, et l'on voit en A une zone de transition semblable à celle qu'on observe dans les tubes de Natterer, mais un peu plus épaisse cependant : la cause de la différence de température étant ici extérieure au tube, il y a évidemment diffusion de la chaleur par les parois.

En inclinant peu à peu le tube, cette zone s'étale et par suite s'amincit, donnant presque l'illusion d'un niveau de liquide.

Laissant la température s'uniformiser dans l'air ambiant à $+18^{\circ}$, le phénomène précédent est encore perceptible au bout d'une heure.

L'expérience réussit encore même si aucun point du tube n'est refroidi au-dessous de $+18^{\circ}$.

Si le tube a été enfermé pendant plusieurs heures dans une armoire où la température soit constante, on ne constate, en le retournant, aucun mouvement dans la masse fluide, et le tube paraît vide; son contenu est alors homogène.

On peut représenter graphiquement les résultats qui précèdent; il convient de tenir compte en même temps des pressions hydrostatiques dues à la pesanteur des fluides, en les exagérant toutefois beau$\operatorname{coup}(1)$.

Sur l'axe d'un tube de Natterer disposé verticalement, considérons une série de masses infiniment petites, et égales entre elles, de liquide ou de vapeur. Le lieu des points figuratifs de ces masses caractérisera l'état du tube à un instant donné ( $f g$. 3 et 4); supposons qu'au début la température soit uniforme, et la saturation réa-

(1) Si l'on voulait représenter à la fois des variations de pression de plusieurs atmosphères et des variations de pression de quelques millimètres d'eau, la figure serait de dimensions inacceptables. 
lisée; le lieu des points figuratifs sera un arc AMNB d'isotherme. Le niveau correspond à la partie $\mathrm{MN}$, et la distance verticale des points A et $B$ représente la différence entre les pressions qui s'exercent aux extrémités du tube. Cette différence est évidemment invariable pour un tube donné.

Quand on élève la température, la vapeur s'échauffe plus vite que le liquide, la ligne figurative devient telle que $\mathrm{A}^{\prime} \mathrm{B}^{\prime}, \mathrm{A}^{\prime}$ et $\mathrm{B}^{\prime}$ étant situés sur deux isothermes différentes.

La ligne $\mathrm{A}^{\prime} \mathrm{B}^{\prime}$ continuant à s'élever devient tangente à la courbe $\mathrm{SS}^{\prime}$ au point critique même, où viennent évidemment se confondre M et $\mathrm{N}$.

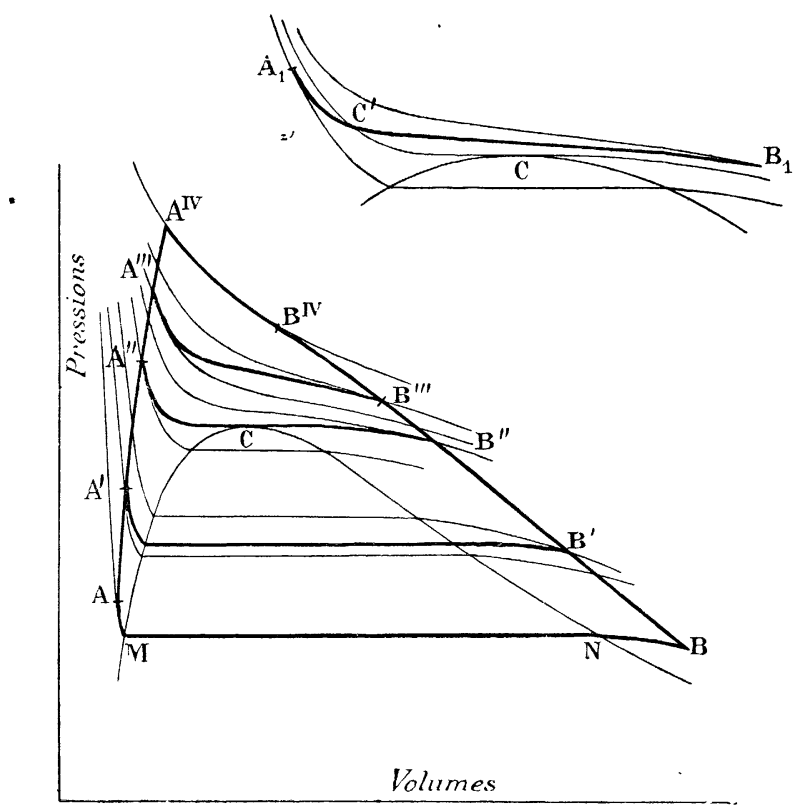

FiG. 3 et 4 .

La température et la pression critique sont réalisées au niveau même, mais la densité ne présente plus de discontinuité quand on passe du liquide à la vapeur, et, par suite, le niveau est rigoureusement invisible.

La forme générale de la ligne $\mathrm{A}^{\prime \prime} \mathrm{CB}^{\prime \prime}$ exprime, conformément à l'observation, que la presque totale variation de la densité se produit sur une hauteur de quelques millimètres, au voisinage du niveau. 
La ligne figurative, continuant à s'élever, conserve la même forme générale, et coupe l'isotherme critique en un point $\mathrm{C}^{\prime}(f i g .4)$ de plus en plus voisin de A. La surface de séparation du liquide et de la vapeur se rapproche de la partie inférieure du tube, mais rien n'indique où elle se trouve à un instant donné, car, suivant une ligne de transformation telle que $A_{1} C^{\prime} B_{1}$, on passe sans transition visible de l'état liquide à l'état gazeux (Andrews).

La température critique éiant dépassée, la ligne figurative devient telle que $\mathrm{A}^{\prime \prime \prime} \mathrm{B}^{\prime \prime}$, et, quand l'équilibre de température est établi, cette ligne se confond avec un arc d'isotherme $\mathrm{A}^{\mathrm{rv}} \mathrm{B}^{\mathrm{rv}}$.

Le lieu des points $\mathrm{AA}^{\prime} \ldots$ et celui de $\mathrm{BB}^{\prime} \ldots$ forment avec $\mathrm{A}^{\mathrm{rv}} \mathrm{B}^{\mathrm{rv}}$ une ligne qui enveloppe la courbe SS'. Dans le cas d'un fluide non pesant, et en supposant la température constamment uniforme et la saturation toujours réalisée, cette ligne se confondrait avec $\mathrm{SS}^{\prime}$ jusqu'à la température pour laquelle le niveau atteindrait l'une des extrémités du tube. On serait dans le cas théorique.

Une discussion très simple montrerait que les lignes $\mathrm{AA}^{\mathrm{Iv}} \mathrm{BB}^{\mathrm{iv}}$ ont bien la forme générale indiquée.

La formule de M. Sarrau (1) permet de calculer, pour l'anhydride carbonique, les différences de densité résultant d'un défaut d'uniformité de la température.

Le calcul, fait par une méthode d'approximations successives, donne les résultats indiqués au tableau suivant: $\mathrm{T}_{c}$ et $\mathrm{V}_{c}$ désignent la température et le volume spécifique critique; $T$ et $V, T^{\prime}$ et $V^{\prime}$, les températures et volumes spécifiques de la vapeur et du liquide. L'instant considéré est celui où le niveau arrive au point critique (courbe $\mathrm{A}^{\prime \prime} \mathrm{CB}^{\prime \prime}$ ).

\begin{tabular}{|c|c|c|c|c|c|}
\hline$\underset{\mathrm{T}}{\mathrm{T}}-\mathrm{T}_{c}$ & $\begin{array}{c}\text { Liquide } \\
\mathbf{T}_{c}-\mathbf{T}^{\prime}\end{array}$ & $\mathbf{T}-\mathbf{T}^{\prime}$ & $\begin{array}{l}\text { Vapeur } \\
\mathrm{V}-\mathrm{V}_{c}\end{array}$ & $\begin{array}{c}\text { Liquide } \\
\mathbf{V}_{c}-\mathbf{V}^{\prime}\end{array}$ & $\mathrm{V}-\mathrm{V}^{\prime}$ \\
\hline $0^{0}, 000302$ & $0^{\circ}, 000306$ & $0^{0}, 000608$ & $2 \% \mathrm{~V}_{c}$ & $2 \% \mathrm{~V}_{c}$ & $4 \% \mathrm{~V}_{c}$ \\
\hline $0^{0}, 00 亏 3$ & $0^{0}, 0062$ & $0^{0}, 0115$ & $5 \%$ & $5 \% "$ & $10 \%$ \\
\hline $0^{0}, 04$ & $0^{0}, 04$ & $0^{0}, 08$ & $10 \%$ & $9 \%$ & $19 \%$ \\
\hline $0^{0}, 04$ & $0^{0}, 054$ & $0^{0}, 094$ & $10 \%$ & $10 \%$ & $20 \% ”$ \\
\hline $0^{0}, 23$ & $0^{0}, 24$ & $0^{0}, 47$ & $20 \%$ & $15 \%$ & $35 \%$ \\
\hline $0^{0}, 23$ & $0^{0}, 43$ & $0^{0}, 66$ & $20 \%$ & $20 \%$ & $40 \%$ \\
\hline
\end{tabular}

Les causes des inégalités de température observées sontles suivantes : $\mathbf{1}^{\circ}$ La masse du liquide est, à volume égal, beaucoup plus grande que celle de la vapeur;

(1) Comptes rendus, t. CI, p. 941 et 1145. 
$2^{\circ}$ La vaporisation est, pour le liquide, une cause de refroidissement;

$3^{\circ}$ La chaleur spécifique du liquide croît avec la température, dépasse celle de l'eau à une distance sensible du point critique, et devient infinie en ce point (1).

\section{Explication des phénomènes présentés par les tubes de Natterer.}

I. - D'après ce qu'on vient de voir, les choses se passent comme si, au point critique, les densités du liquide et de la vapeur étaient différentes. Or, dans un tube où la densité moyenne est comprise entre celle du liquide et celle de la vapeur, le niveau existe; par suite, le niveau pourra être observé, jusqu'à ce qu'il arrive à la température critique, avec des tubes dans lesquels la densité moyenne peut varier dans le rapport de 10 à 15 .

De plus, le niveau doit peu se déplacer pendant l'échauffement. Si la densité moyenne est inférieure à la densité critique, la vapeur occupe plus de la moitié du tube; comme elle se surchauffe et demeure constamment moins dense que si elle était saturée à la température du niveau, le liquide a beaucoup moins de vapeur à fournir que si la saturation était réalisée. Au point de vue de la variation de volume, la dilatation du liquide compensera en grande partie la vaporisation.

Si la densité moyenne dépasse la densité critique, le niveau doit s'élever, parce que le liquide se dilate et par suite de la condensation finalement totale de la vapeur. Or, cette condensation ne se produit pas, la vapeur étant surchauffée, et l'une des deux causes de l'ascension du niveau est supprimée.

II. - Quand le niveau arrive à la température critique, la pression au niveau même est nécessairement égale à la pression critique ( $f g .3)$; elle est, aux effets de pesanteur près, la même dans tout le tube et, par suite, est indépendante du remplissage dans les limites où le niveau est observable jusqu'au point critique. Ce résultat est conforme à l'observation.

III. - En arrivant à la température critique, le niveau disparaît dans une couche de transition où la densité varie d'une manière continue (effets de mirage). Ce phénomène, dont on a vu l'explication,

(1) Il résulte des expériences faites par M. Mathias (C.R., t. CIX, p. 404) qu'à $0^{\circ}, 5$ au-dessous de la température critique la chaleur spécifique de l'anhydride sulfureux liquide est sensiblement triple de celle de l'eau. 
se produira toujours quand on atteindra le point critique par élévation de la température. L'échauffement ne pouvant être infiniment lent, et la chaleur spécifique du liquide devenant infinie, des inégalités de température se produiront nécessairement, ayant leur maximum d'effet au point critique, où le coefficient de dilatation à pression constante devient infini.

Cette couche de transition ne saurait être considérée comme étant un état transitoire du ménisque. La fig. 4 montre, en effet, que le niveau $\mathrm{C}^{\prime}$ descend progressivement vers le bas du tube, en restant complètement invisible, et abandonne la zone de transition. Celle-ci; constituée alors par du gaz, marque seulement la région du tube où se sont produites les inégalités de température.

La formation de stries, au voisinage du lieu de disparition du niveau, s'explique aisément si l'on considère que, d'après le tableau précédent, de très faibles différences de température suffisent à produire de notables variations de densité.

IV. - Il est évident que, si on agite fréquemment les tubes pendant l'échauffement, les inégalités de température disparaîtront presque complètement, ainsi que les phénomènes qui en dépendent. Il en sera de même si on atteint la température critique par refroidissement.

L'influence de la pesanteur produit des effets analogues aux précédents, auxquels ils s'ajoutent, mais beaucoup plus faibles.

Étant donné un tube de Natterer, maintenu à la température critique, la ligne figurative de l'état du tube, si l'on tient compte de la pesanteur, est un.arc de l'isotherme critique, et comprend le point critique pour un remplissage convenable. Au voisinage du niveau critique, où la compressibilité devient infinie, la variation du volume spécifique atteint, d'après les calculs de M. Gouy $\left({ }^{1}\right), 20 / 0$ du volume critique sur une hauteur de $3^{\mathrm{mm}}, 86$ et $40 / 0$ sur une hauteur de $30^{\mathrm{mm}}, 96$.

Mais les variations correspondantes de l'indice sont très faibles, même pour la hauteur de $30^{\mathrm{mm}}, 96$, qui dépasse celle de la zone de transition ordinairement observée. En effet, l'indice de l'anhydride carbonique liquide est seulement 1,196 à $+15^{\circ}$ (Bleekrode), et l'indice critique est 1,12 , si on admet la constance du rapport $\frac{n-1}{\mathrm{D}} ;$ à des variations de volume spécifique de $20 / 0$ et $40 / 0$ correspondent

(1) Comptes rendus, t. CXV, p. 720. 
par suite des variations d'indice égales à 0,0024 et 0,0048 . Pour cette dernière valeur, l'écartement apparent de deux génératrices extérieures du tube ne varie, par l'effet de la réfraction, que de $10 / 0$ sur la hauteur de $30^{\mathrm{mm}}, 96$ considérée. La variation de l'écartement est au contraire de $60 / 0\left(^{4}\right)$, et peut être appréciable pour une variation de volume spécifique de $200 / 0$, correspondant, ainsi qu'on l'a $\mathrm{vu}$, à une différence de température de $0^{\circ}, 094$, très inférieure à celles observées dans les expériences I et III. Une différence de température de $0^{\circ}, 0006$ suftirait, d'ailleurs, à produire le même effet que la pesanteur agissant sur une hauteur de $30^{\mathrm{mm}}, 96$.

Il semble donc que le défaut d'uniformité de la température soit la cause principale des phénomènes observés. 